\title{
Multifunctional Nanostructured Materials Applied in Controlled Radiopharmaceuticals Release
}

\author{
Raquel Cristina de Sousa Azevedo ${ }^{1}$, Daniel Crístian Ferreira Soares ${ }^{1}$, Ricardo Geraldo de Sousa ${ }^{2}$, \\ Edésia Martins Barros de Sousa ${ }^{{ }^{*}}$
}

${ }^{1}$ Centro de Desenvolvimento da Tecnologia Nuclear (CDTN)/CNEN, Serviço de Nanotecnologia, Belo Horizonte, Brazil; ${ }^{2}$ Departamento de Engenharia Química, Universidade Federal de Minas Gerais, Belo Horizonte, Brazil.

Email: *sousaem@cdtn.br

Received January $6^{\text {th }}, 2012$; revised February $24^{\text {th }}, 2012$; accepted March $13^{\text {th }}, 2012$

\begin{abstract}
The metaiodobenzylguanidine (MIBG) radiopharmaceutical, an analogue of norepinephrine, has been used to diagnose certain diseases in the cardiovascular system when radiolabeled with ${ }^{123}$ I. This radiopharmaceutical can also be used to treat tumors, such as neuroblastomas and pheochromocytomas, when radiolabeled with ${ }^{131} \mathrm{I}$. Its clinical use is often accompanied by a slow intravenous administration, where a significant dose of radiation can directly affect workers in nuclear medicine services. To overcome this problem, the incorporation and controlled release of radiopharmaceuticals from the matrix of mesoporous systems based on silica, such as SBA-15 and hybrid [SBA-15/P(N-iPAAm)], can lead to a significant reduction in radiation doses received by workers. In the present study, silica matrices SBA-15 and hybrid [SBA-15/P $(N$-iPAAm $)]$ containing the radiopharmaceutical MIBG were prepared and physicochemically characterized through FTIR, SEM, and small angle X-ray diffraction techniques. The release profiles of MIBG from SBA-15 and [SBA-15/P( $N$-iPAAm)] were studied in a simulated body fluid (SBF) to evaluate their potential application as vehicles for controlled releases. Furthermore, in vitro studies were performed to assess the cytotoxicity of matrices as compared to human lung fibroblast cells (MRC-5). The results revealed that the amount of MIBG incorporated within the studied matrices was indeed quite different, showing that only the hybrid [SBA-15/P $(N$-iPAAm $)]$ system allowed for a more adequate release profile of MIGB. Taking all results into consideration, it can be concluded that the hybrid matrix [SBA-15/P $(N-$ iPAAm $)]$ can be considered a potential alternative material for the controlled release delivery of radiopharmaceuticals.
\end{abstract}

Keywords: SAB-15; Hybrid [SBA-15/P(N-iPAAm)]; MIGB Radiopharmaceutical; Drug Release

\section{Introduction}

The Metaiodobenzylguanidine (MIBG) radiopharmaceutical is an analogue of norepinephrine, which competes for capture, accumulation, and release processes in adrenergic nerve endings. MIBG is not metabolized by Catechol-O-Methyl-Transferase (COMT) and Monoamine Oxidase (MAO), which, once radiolabeled with ${ }^{123} \mathrm{I}$, allows for its use in the noninvasive evaluation of the adrenergic activity of the cardiovascular system, providing valuable prognostic information [1-4]. Furthermore, MIBG can be used in tumor treatments, such as neuroblastomas and pheochromocytomas, when radiolabeled with ${ }^{131} \mathrm{I}$ [5-7]. The clinical use of MIBG is often accompanied by a slow intravenous administration, where a significant dose of radiation can directly affect workers in nuclear medicine services [8-10].

One means through which to solve this problem is the

"Corresponding author. use of implants composed of materials that can promote the controlled release of the radiopharmaceutical, thus avoiding the presence of workers during the infusion process. In this context, ordered mesoporous materials have been the subject of a growing number of studies in different applications, including the controlled release of drugs $[11,12]$. The mesoporous silica with hexagonal structure, SBA-15, presents an ordered hexagonal arrangement of unidirectional mesoporous channels containing a diameter of approximately 6 to $10 \mathrm{~nm}$; a high surface area of above $800 \mathrm{~m}^{2} / \mathrm{g}$, depending on the synthesis conditions; and good thermal and hydrothermal stability $[13,14]$. Due to these structural characteristics, SBA-15 materials present a high potential for the incorporation and release of a wide variety of molecules (organic and inorganic) that can provide therapeutic activities $[15,16]$.

Temperature-responsive hydrogels, such as $\operatorname{poly}(N$ isopropylacrylamide) $\mathrm{P}(\mathrm{N}$-iPAAm), are also a well-studi- 
ed class of drug delivery systems, as they can respond pronouncedly to temperature changes [17-20]. In water, $\mathrm{P}(\mathrm{N}$-iPAAm) exhibits a phase transition at a lower critical solution temperature (LCST) of approximately $33^{\circ} \mathrm{C}$. Below the LCST, the hydrogel incorporates water and swells, whereas the release of water in response to an increase in temperature causes shrinkage $[21,22]$.

Therefore, the combination of the mesoporous material SBA-15 with the polymeric gel poly( $N$-isopropylacrylamide) can lead to the formation of a hybrid material with a potential for application as new drug delivery systems, given that self-regulated delivery allows for drug release when needed [23].

Some studies on hybrid systems based on $\mathrm{P}(\mathrm{N}$-iPAAm $)$ and ordered mesoporous materials have been reported in the literature and have shown interesting results [24-26]. Studies have found that the resultant hybrid systems, as compared to pure polymers, exhibit an increased mechanical and chemical performance and even unique properties due to their regular mesoscopic structure and space restriction effect $[17,27]$. In this sense, the incorporation of the polymer phase into mesoporous silica can led to a significant change in the structural properties of the system without destroying the ordered hexagonal structure of SBA-15, which makes this system promising for a variety of intelligent applications, especially in the controlled release of the radiopharmaceutical.

Considering that the special properties of these hybrid systems are useful in various structural and biomedical applications due to their specific characteristics, the present work aimed to evaluate a special synthesis route to obtain [SBA-15/P( $N$-iPAAm)]. This study investigated the cytotoxicity of both samples by MTT assay, as well as the influence of the presence of a polymeric species within SBA-15 on the behavior of silica matrices as a controlled drug release device, and compared the release kinetics of the model drug (MIBG) from pure SBA-15.

\section{Experimental}

\subsection{Synthesis}

SBA-15 silica was prepared according to Zhao [14] using commercial triblock copolymer Pluronic P123-PEO20PPO70-PEO20 [poly(ethylene glycol)-block-poly (propylene glycol)-block-poly(ethylene glycol)] (Sigma-Aldrich) as a templating agent. The P123 copolymer was dissolved in a mixture of distilled water and $\mathrm{HCl}$ under stirring, followed by the addition of tetraethyl orthosilicate (TEOS). The mixture was maintained at $35^{\circ} \mathrm{C}$ for 24 $\mathrm{h}$, and then for an additional $24 \mathrm{~h}$ at $100^{\circ} \mathrm{C}$ under static conditions in a Teflon-lined autoclave. The obtained material was filtered and dried at $40^{\circ} \mathrm{C}$ and the surfactant was removed by calcination $\left(550^{\circ} \mathrm{C}\right.$ for $\left.5 \mathrm{~h}\right)$.

The hybrid was prepared using the following proce- dure [28]: $0.5 \mathrm{~g}$ of calcined SBA-15 was added to a 3.5 $\mathrm{mL}$ solution of $0.245 \mathrm{~g}$ of monomer ( $\mathrm{N}$-isopropylacrylamide $-N$-iPAAm) and $0.005 \mathrm{~g}$ of crosslinking agent (N,N,N', N'-methylene-bisacrylamide-MBA). The mixture was transferred to an INNOVA 4200 (150 rpm) shaker, and the mixture was continuously purged with nitrogen. The solution was then allowed to polymerize for $24 \mathrm{~h}$ in a water bath at $10^{\circ} \mathrm{C}$. The obtained hybrid material was dried at $60^{\circ} \mathrm{C}$ for $24 \mathrm{~h}$ and subsequently washed to remove the excess of monomers, crosslinking agent, initiator, and accelerator. In the washing stage, the material was disaggregated, suspended in water, and continually stirred. The hybrid was then collected by centrifugation at $3600 \mathrm{rpm}$ for $3 \mathrm{~min}$ and dried at $60^{\circ} \mathrm{C}$ for $24 \mathrm{~h} \mathrm{[29].}$

\subsection{Characterization}

The FTIR measurements were conducted with an ABB Bomen model MB102 spectrophotometer within the range of $4000-400 \mathrm{~cm}^{-1}$. The FTIR spectra were recorded at room temperature using $\mathrm{KBr}$ pellets and a resolution of $4 \mathrm{~cm}^{-1}$ and $64 \mathrm{scans} / \mathrm{min}$. SEM characterization was performed in a scanning electron microscope (Quanta 200 FEG-FEI) operating at $30 \mathrm{kV}$. The small-angle XRD patterns were obtained using an Ultima IV ${ }^{\circledR}$ (Rigaku Inc.) $3 \mathrm{~kW}$ generator rotating equipment anode $\mathrm{X}$-ray diffraction equipped with a copper anode $(\lambda$ $=1.54 \AA \hat{A})$. The generator was operated at $40 \mathrm{kV}$ and 30 $\mathrm{mA}$. The incident X-ray was set at a wavelength of 1.488 $\AA$, while the angle scattering $2 \theta$ ranged from $0^{\circ}$ to $5^{\circ}$.

\subsection{MIBG Loading and in Vitro Release Study}

SBA-15 and [SBA-15/P( $N$-iPAAm)] were loaded with Metaiodobenzylguanidine (MIBG) as follows: $0.2 \mathrm{~g}$ of the powder sample was added to $25 \mathrm{~mL}$ of an MIBG solution $\left(800 \mu \mathrm{g} \cdot \mathrm{mL}^{-1}\right)$ and shaken for $48 \mathrm{~h}$ at $25^{\circ} \mathrm{C}(200$ $\mathrm{rpm}$ ). A 1:10 weight ratio of the MIBG/solid sample was used. Powder MIBG-loaded samples were recovered by filtration, washed with distilled water, and left to dry for $24 \mathrm{~h}$ at $60^{\circ} \mathrm{C}$. The in vitro release profile was obtained by soaking the samples $(50 \mathrm{mg})$ in $30 \mathrm{~mL}$ of a simulated body fluid (SBF) [30] for 24hs at room temperature. The UV spectrometry procedure (Shimadzu UV-VIS V-V 2550) was used to monitor the amount of MIBG that had been loaded and released as a function of time. The solutions were continuously stirred, and the concentration of MIBG in SBF was determined by the intensity of the absorption band at $230 \mathrm{~nm}$.

\subsection{Cytotoxicity Study}

The cytotoxicity of SBA-15 and [SBA-15/P(N-iPAAm)] were evaluated by means of MTT ((3-(4,5-Dimethylthiazol-2-yl)-2,5-diphenyltetrazolium bromide) assay in hu- 
man lung fibroblast cells (MRC-5), obtained from the radiobiology laboratory from the Center for the Development of Nuclear Technology (CDTN/CNEN).

The cells ( 1500 cells/well) were subjected to treatment for 48 hours, under increasing concentrations of SBA-15 and [SBA-15/P(N-iPAAm)] $\left(0.1\right.$ to $\left.200 \mu \mathrm{g} \cdot \mathrm{mL}^{-1}\right)$. Eight replicates were investigated for statistical evaluation. The determination of the absorbance at $570 \mathrm{~nm}$ of the formed product is a measure of metabolic cell viability, and both quantities are directly proportional.

\subsection{Statistics}

All experiments were performed in triplicate and expressed as the mean \pm standard deviation, unless otherwise stated. The specific activity data were compared by means of the Student's t-test, using the prism 4.0 software, considering a $95 \%$ confidence interval.

\section{Results and Discussion}

\subsection{Synthesis}

Figure 1 shows the FTIR spectra of all studied samples. The spectra of pure SBA-15 and the hybrid sample exhibited different absorption bands. The SBA-15 spectrum was dominated by $\mathrm{O}-\mathrm{H}$ modes, presenting a broad and intense band at $3440 \mathrm{~cm}^{-1}$ assigned to hydroxyl groups. A broad and very intense band in the range $1000-1200$ $\mathrm{cm}^{-1}$, corresponding to $\mathrm{Si}$-O-Si modes of the SBA-15 siliceous matrix, was also present. The band at $960 \mathrm{~cm}^{-1}$ could be assigned to $\delta(\mathrm{HOH})$ physisorbed water and the $\mathrm{Si}-\mathrm{O}$ stretching vibration of surface $\mathrm{Si}-\mathrm{OH}$ groups. In hybrid systems, the bands corresponding to the $\mathrm{l}$ - $\mathrm{H}$ modes of P(N-iPAAm) are commonly observed between 2972 and $2875 \mathrm{~cm}^{-1}$; the bands attributed to the isopropyl group were located at 1386 and $1368 \mathrm{~cm}^{-1}$; the band corresponding to the bending vibration of $\mathrm{CH}_{3}$ was located at $1456 \mathrm{~cm}^{-1}$; while the bands arising from $\mathrm{C}=\mathrm{O}$ stretching and $\mathrm{N}-\mathrm{H}$ bending vibrations were observed at 1645 and $1550 \mathrm{~cm}^{-1}$, respectively.

The monomer characteristic bands $\left(L C=C 1620 \mathrm{~cm}^{-1}\right.$; $\delta \mathrm{CH}_{2}=1409 \mathrm{~cm}^{-1} ; \delta \mathrm{H}_{2} \mathrm{C}=\mathrm{C}-1305$ and $1325 \mathrm{~cm}^{-1} ; \delta$ vinyl group at 990 and $916 \mathrm{~cm}^{-1}$ ) were not present in the hybrid sample spectra, as can be seen in the scale-expanded FTIR spectrum in the $1800-900 \mathrm{~cm}^{-1}$ region (Figure 2). These results prove the presence of $\mathrm{P}(\mathrm{N}$-iPAAm $)$ in SBA15 pores with no other significant synthesis components (monomer, initiator, or accelerator) and demonstrate the successful conversion of the monomers into polymer [29].

Figure 3 shows SEM images of the SBA-15 and [SBA-15/P( $N$-iPAAm $)]$ samples. SBA-15 consists of many rope-like domains with average sizes of $1.7 \mu \mathrm{m}$ aggregated into wheat-like macrostructures, Figure 3(a). A

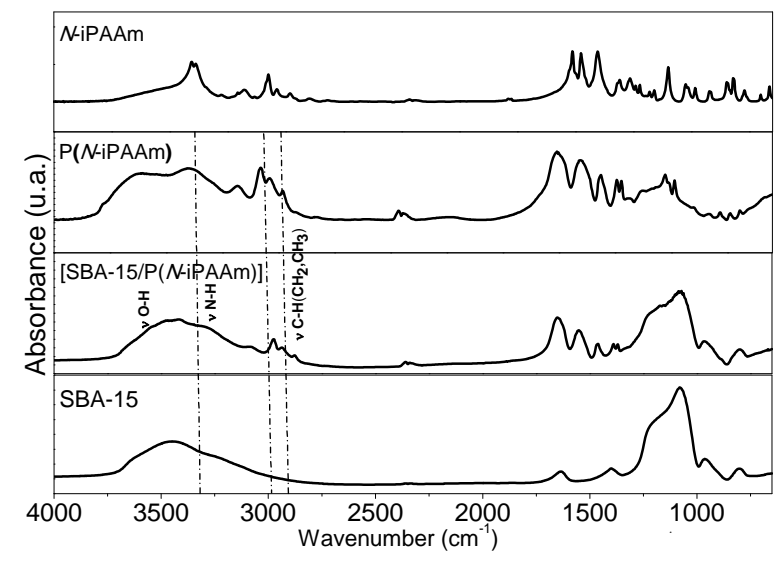

Figure 1. FTIR spectra of $N$-iPAAm, P( $N$-iPAAm), [SBA15/P( $N$-iPAAm $)$, and SBA-15.

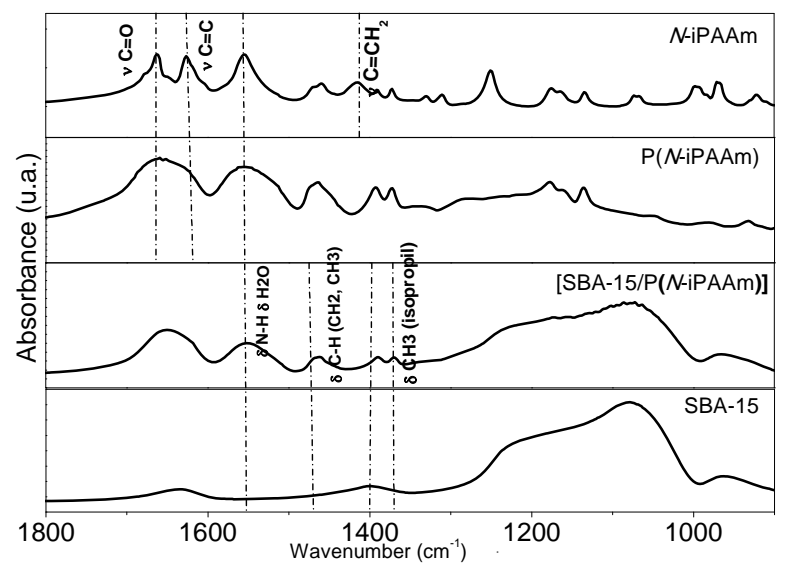

Figure 2. FTIR spectra of $N$-iPAAm, P( $N$-iPAAm), [SBA-15/ $\mathrm{P}(\mathrm{N}$-iPAAm $)]$, and SBA-15 expanded in the range of 1800 $900 \mathrm{~cm}^{-1}$.

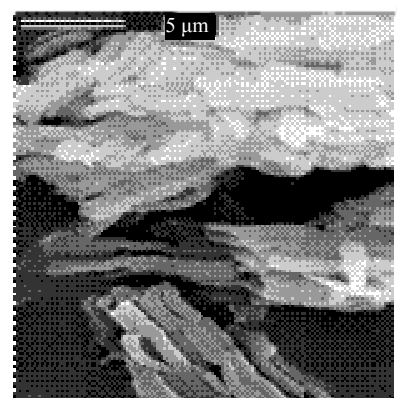

(a)

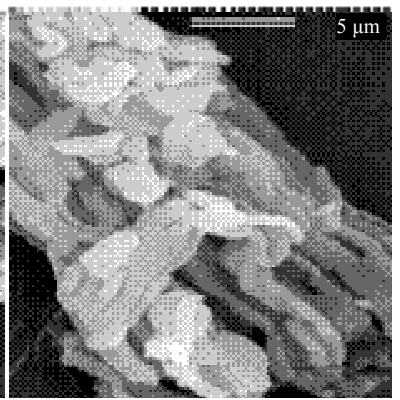

(b)
Figure 3. SEM micrographs of (a) SBA-15 and (b) [SBA15/P( $N$-iPAAm)] hybrid samples.

similar morphology was observed after the polymerizetion of $\mathrm{P}(N$-iPAAm $)$ within the SBA-15 network, presenting a $450 \mathrm{~nm}$ width (Figure 3(b)), indicating that the incorporation of the hydrogel did not change this typical characteristic of mesoporous silica.

Figures 4(a) and (b) show the profile at a low angle for pure SBA-15 and [SBA-15/P(N-iPAAm)], respectively. 


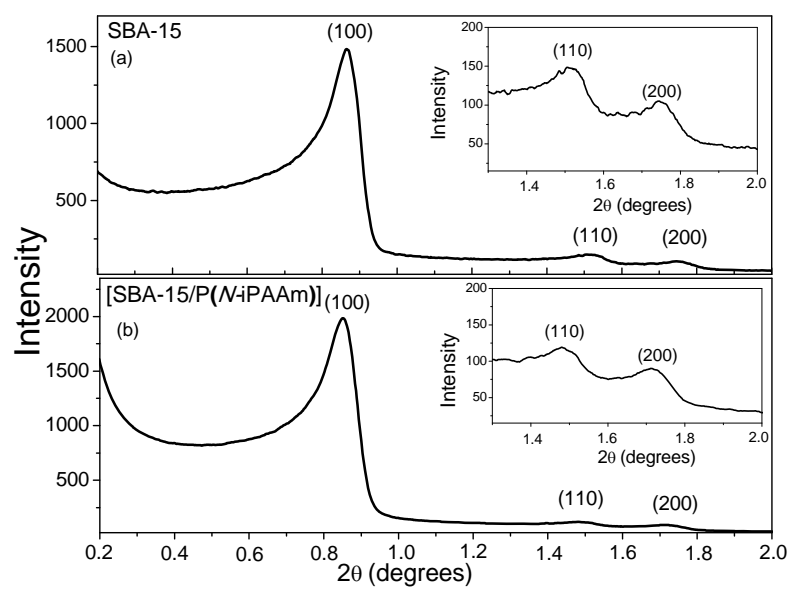

Figure 4. Small-angle XRD patterns of SBA-15 (a) and [SBA-15/P(N-iPAAm)] (b).

The three diffraction peaks of the former, at $2 \theta=0.87$, 1.51 , and 1.75 , can be indexed as ( $\left.\begin{array}{lll}1 & 0 & 0\end{array}\right),\left(\begin{array}{lll}1 & 1 & 0\end{array}\right)$, and ( 2 $00)$ reflections, respectively, which are typical of hexagonally structured SBA-15 silica with highly or- dered mesoporous channels. A shift of these diffraction peaks to smaller $2 \theta$ values was identified for [SBA-15/ $\mathrm{P}(N$ iPAAm)] (Figure 4(b)), possibly due to the formation of the hydrogel phase within the silica channels. The values of interplanar spacing, $\mathrm{d}(100)$, and the unit cell parameter, $\mathrm{a}_{100}$, found for the SBA-15 and [SBA-15/ P(N-iPAAm)] samples are reported in Table 1.

\subsection{Drug Loading and Release Study}

The uptake of MIBG by different samples was determined by UV-Vis. Table 2 shows this radiopharmaceutical content for SBA-15 and [SBA-15/P( $N$-iPAAm)] samples. The results reveal that the hybrid system sample presents a higher amount of the incorporated drug, which is likely related to a greater available interaction among them.

The in vitro MIBG release properties from these samples were investigated as a function of time and are

Table 1. Structural parameters of SBA-15 and [SBA-15/ P(N-iPAAm)] samples.

\begin{tabular}{cccc}
\hline Sample & $2 \theta$ & $\mathrm{d}_{100}(\mathrm{~nm})$ & $\mathrm{a}_{100}(\mathrm{~nm})$ \\
\hline SBA-15 & 0.87 & 10.16 & 11.74 \\
{$[\mathrm{SBA}-15 / \mathrm{P}(N$-iPAAm) $]$} & 0.85 & 10.39 & 12.00 \\
\hline
\end{tabular}

Table 2. Loading rate of MIBG into SBA-15 and [SBA15/P(N-iPAAm)] samples.

\begin{tabular}{cc}
\hline Sample & Quantity Incorporated $(\% \mathrm{~m} / \mathrm{m})$ \\
\hline SBA-15 & 14 \\
{$[$ SBA-15/P $(N$-iPAAm $)]$} & 65 \\
\hline
\end{tabular}

shown in Figure 5. It could be observed that the MIBGloaded SBA-15 sample did not show a sharp initial burst release during the first hours. By contrast, the release profiles of [SBA-15/P $(N$-iPAAm) $]$ exhibited a pronounced initial burst release effect of $70 \%$, followed by a slow release pattern. The initial burst release can be attributed to the immediate dissolution and release of the portion of the drug located on or near the surface of the disks. It could also be observed that the SBA-15 matrix released a smaller percentage of MIBG than did hybrid samples.

To investigate more precisely the effect of the mesoporous network due to the presence of a hydrogel on the release of MIBG, the results were analyzed according to the Higuchi model [31], an empirical exponential expression used to relate drug release with fractional release time (Equation 1):

$$
M t / M \infty=K t^{n}
$$

where $M t / M \infty$ is the fractional solute release, $t$ is the release time, $K$ is a constant, and $n$ is the diffusional exponent characteristic of the release mechanism.

A linear regression was used to fit the data. The 0.5 diffusional exponent in the plot indicates a predominantly diffusional control with regard to the leaching of the drug into the immersion fluid. The drug is presumed to dissolve slowly into the fluid phase and to diffuse from the system along the solvent-filled capillary channels. The values of the kinetic constant $k$ for different samples are presented in Table 3.

Comparing the results, the [SBA-15/P $(N$-iPAAm $)]$ sample presented a higher level of MIBG release kinetics. Differences in microstructure are suggested to explain these behaviors. It has been previously suggested that structural mesopores can present micropores, depending on synthesis parameters [27]. In this sense, it can be suggested that the favorable release kinetic of MIBG from

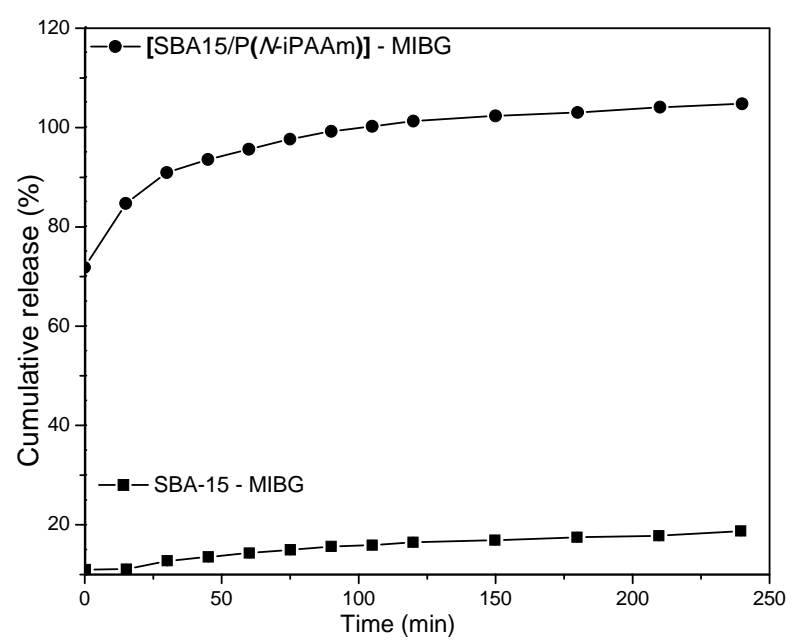

Figure 5. Release profiles of MIBG from SBA-15 and [SBA15/P( $N$-iPAAm)] samples. 
Table 3. Kinetic parameters.

\begin{tabular}{ccc}
\hline Sample & $\mathrm{K}$ & $\mathrm{r}^{2}$ \\
\hline SBA-15 & 3.3 & 0.99 \\
{$[$ SBA-15/P $(N$-iPAAm $)]$} & 51.5 & 0.98 \\
\hline
\end{tabular}

the $[\mathrm{SBA}-15 / \mathrm{P}(N$-iPAAm $)]$ system is due to the absence of microporosity in the structure of these samples. Therefore, the slower release of MIBG from pure SBA15 can be attributed to the presence of micropores in the structure of these samples, which may well influence the release kinetics. A second possibility suggests the interaction between radiopharmaceuticals and the mesoporous silica through hydrogen bonds due to the affinity of MIBG molecules and silanol groups present in the mesoporous silica.

\subsection{Cytotoxicity Study}

The cytotoxicity profile of a new material should be investigated since undesirable side effects can occurs when this material is used in in vivo applications. The cytotoxicity of the hybrid [SBA-15/P(N-iPAAm)] and SBA 15 were evaluated through MTT assay, and the results showed that the groups of cells exposed to SBA-15 and [SBA$15 / \mathrm{P}(N$-iPAAm $)]$, in all tested concentrations, presented a viability of approximately $100 \%$ (Figure 6).

The MTT assay evaluates the ability of the enzyme succinate dehydrogenase to reduce the MTT substrate in formazan crystals through mitochondrial activity. Thus, the metabolic activity of the cells can be evaluates. The obtained results indicate, even in high concentrations, that both studied materials are not harmful to normal body cells. However, more detailed studies should be conducted to confirm the biocompatibility of these materials.

\section{Conclusion}

Mesoporous silica SBA-15 was synthesized in a hybrid system using $\mathrm{P}(\mathrm{N}$-iPAAm $)$ as an organic phase and incorporated within MIBG to establish the influence of the presence of an polymeric species within the SBA-15 structure on controlled radiopharmaceutical release. Characterization shows that the properties of the formed solids are highly influenced by the presence of hydrogels. The results indicate that the $[\mathrm{SBA}-15 / \mathrm{P}(N$-iPAAm $)]$ hybrid-ordered mesopores present a potential to encapsulate bioactive molecules and demonstrate that MIBG can be incorporated in significant amounts within studied matrices. However, only SBA-15/P(N-iPAAm) hybrid system allowed for a more adequate release profile of MIGB.

\section{Acknowledgements}

This research was supported by the Brazilian agencies

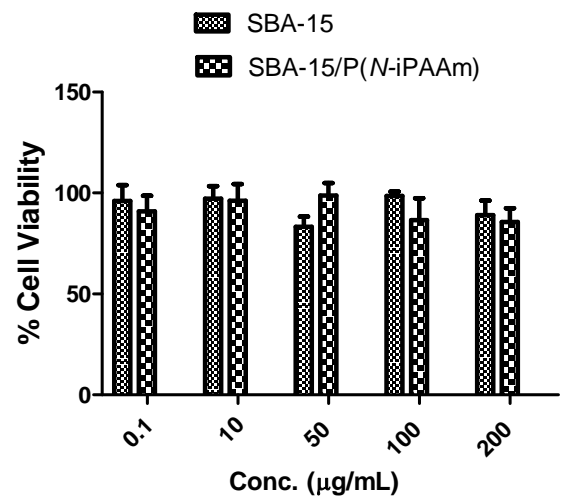

Figure 6. Viability of MRC-5 cells in different concentrations of SBA 15 and [SBA-15/P( $N$-iPAAm)] treated for 48 hours. The results showed no statistically significant difference $(p>0.05)$.

CAPES, CNPq, and FAPEMIG. The authors would like to thank the Microscopy Center at UFMG for its technical support during electron microscopy work.

\section{REFERENCES}

[1] J. W. Ha, J.-D. Lee, Y. Jang, N. Chung, J. Kwan, S.-J. Rim, Y.-J. Lee and S.-S. Kim, "123I-MIBG Myocardial Scintigraphy as a Noninvasive Screen for the Diagnosis of Coronary Artery Spasm," Journal of Nuclear Cardiology, Vol. 5, No. 6, 1998, pp. 591-597. doi:10.1016/S1071-3581(98)90113-1

[2] H. Ogita, T. Shimonagata, M. Fukunami, et al., "Prognostic Significance of Cardiac ${ }^{123}$ I Metaiodobenzylguanidine Imaging for Mortality and Morbidity in Patients with Chronic Heart Failure: A Prospective Study," Heart, Vol. 86, No. 6, 2001, pp. 656-660. doi:10.1136/heart.86.6.656

[3] D. Agostini, I. Carrió and H. J. Verbene, "How to Use Myocardial ${ }^{123}$ I-MIBG Scintigraphy in Chronic Heart Failure," European Journal of Nuclear Medicine and Molecular Imaging, Vol. 36, No. 4, 2009, pp. 555-559. doi:10.1007/s00259-008-0976-X

[4] A. F. Jacobson, J. Lombard, G. Banerjee, et al., " ${ }^{123} \mathrm{I}-$ MIBG Scintigraphy to Predict Risk for Adverse Cardiac Outcomes in Heart Failure Patients: Design of Two Prospective Multicenter International Trials," Journal of $\mathrm{Nu}$ clear Cardiology, Vol. 16, No. 1, 2009, pp. 113-121. doi:10.1007/s12350-008-9008-2

[5] K. S. S. Bhati, M. M. Ismail, A. Sahdev, A. G. Rockall, K. Hogarth, A. Canizales, N. Avril, J. P. Monson, A. B. Grossman and R. H. Reznek, "123 I-Metaiodobenzylguanidine (MIBG) Scintigraphy for the Detection of Adrenal and Extra-Adrenal Phaeochromocytomas: CT and MRI Correlation," Clinical Endocrinology, Vol. 69, No. 2, 2008, pp. 181-188. doi:10.1111/j.1365-2265.2008.03256.x

[6] B. Rose, K. K. Matthay, D. Price, J. Huberty, B. Klencke, J. A. Norton, et al., "High-Dose 131I Metaiodobenzylguanidine Therapy for 12 Patients with Malignant Pheochromocytoma," Cancer, Vol. 98, No. 2, 2003, pp. 239248. doi:10.1002/cncr.11518 
[7] J. Rob, Mairs, Boyd, "Optimizing MIBG Therapy of Neuroendocrine Tumors: Preclinical Evidence of Dose Maximization and Synergy," Nuclear Medicine and Biology, Vol. 35, No. 1, 2008, pp. 9-20. doi:10.1016/j.nucmedbio.2008.04.008

[8] J. B. Bomanji, W. Wong, M. N. A. Gaze, W. Cassoni, J. S. Waddington and P. J. Ell, "Treatment of Neuroendocrine Tumours in Adults with ${ }^{131}$ I-MIBG Therapy," Clinical Oncology, Vol. 15, No. 4, 2003, pp. 193-198. doi:10.1016/S0936-6555(02)00273-X

[9] F. Giammarile, A. Chiti, M. Lassmann, B. Brans and G. Flux, "EANM Procedure Guidelines for ${ }^{131}$ I-Meta-Iodobenzylguanidine ( $\left.{ }^{131} \mathrm{I}-\mathrm{mIBG}\right)$ Therapy," European Journal of Nuclear Medicine and Molecular Imaging, Vol. 35, No. 5, 2008, pp. 1039-1047. doi:10.1007/s00259-008-0715-3

[10] L. Troncone and V. Rufini, "131 I-MIBG Therapy of Neural Crest Tumours (Review)," Anticancer Research, Vol. 17, No. 3, 1997, pp. 1823-1831.

[11] A. Taguchi and F. Schüth, "Ordered Mesoporous Materials in Catalysis," Microporous and Mesoporous Materials, Vol. 77, No. 1, 2005, pp. 1-45. doi:10.1016/j.micromeso.2004.06.030

[12] S. Wang, "Ordered Mesoporous Materials for Drug Delivery," Microporous and Mesoporous Materials, Vol. 117, No. 1-2, 2009, pp. 1-9. doi:10.1016/j.micromeso.2008.07.002

[13] U. Ciesla and F. Schüth, "Ordered Mesoporous Materials," Microporous and Mesoporous Materials, Vol. 27, No. 2-3, 1999, pp. 131-149. doi:10.1016/S1387-1811(98)00249-2

[14] D. Zhao, Q. Huo, J. Feng, B. F. Chmelka and G. D. Stucky, "Nonionic Triblock and Star Diblock Copolymer and Oligomeric Surfactant Syntheses of Highly Ordered, Hydrothermally Stable, Mesoporous Sílica Structures," Journal of the American Chemical Society, Vol. 120, No. 24, 1998, pp. 6024-6036. doi:10.1021/ja974025i

[15] J. S. Beck and J. C. Vartuli, "Recent Advances in the Synthesis, Characterization and Applications of Mesoporous Molecular Sieves," Current Opinion in Solid State \& Materials Science, Vol. 1, No. 1, 1996, pp. 76-87. doi:10.1016/S1359-0286(96)80014-3

[16] M. Hartmann, "Ordered Mesoporous Materials for Bioadsorption and Biocatalysis," Chemistry of Materials, Vol. 17, No. 18, 2005, pp. 4577-4593. doi: $10.1021 / \mathrm{cm} 0485658$

[17] D. C. Coughlan, F. P. Quilty and O. I. Corrigan, "Effect of Drug Physichochemical Properties on Swelling/Deswelling Kinetics and Pulsatile Drug Release from Thermoresponsive Poly( $N$-isopropylacrylamide) Hydrogels," Journal of Controlled Release, Vol. 98, No. 1, 2006, pp. 97-114. doi:10.1016/j.jconrel.2004.04.014

[18] A. S. Hoffman, "Applications of Thermally Reversible Polymers and Hydrogels in Therapeutics and Diagnostics," Journal of Controlled Release, Vol. 6, No. 1, 1987 , pp. 297-305. doi:10.1016/0168-3659(87)90083-6

[19] N. A. Peppas, "Hydrogels in Pharmaceutical Formulations," European Journal of Pharmaceutics and Biopharmaceutics, Vol. 50, No. 1, 2000, pp. 27-46.

\section{doi:10.1016/S0939-6411(00)00090-4}

[20] N. A. Peppas and R. Langer, "Advances in Biomaterials, Drug Delivery, and Bionanotechnology," Bioengineering, Food, and Natural Products, Vol. 49 No. 12, 2003, pp. 2990-3006. doi:10.1002/aic.690491202

[21] R. F. S. Freitas and E. L. Cussler, "Temperature Sensitive Gels as Extraction Solvents," Chemical Engineering Science, Vol. 42, No. 1, 1987, pp. 97-103. doi:10.1016/0009-2509(87)80213-0

[22] R. F. S. Freitas and E. L. Cussler, "Temperature Sensitive Gels as Size Selective Absorbants," Separation Science and Technology, Vol. 22, No. 2-3, 1987, pp. 911-919. doi:10.1080/01496398708068989

[23] V. Castelvetro and C. De Vitaa, "Nanostructured Hybrid Materials from Aqueous Polymer Dispersions," Advances in Colloid and Interface Science, Vol. 108-109, 2004, pp. 167-185. doi:10.1016/j.cis.2003.10.017

[24] Y. Chun and B.-S. Tian, "Preparation and Characterization of Thermo-Sensitive Mesoporous PNIPAAm/SBA15 Composite," Acta Chimica Sinica, Vol. 66, No. 5, 2008, pp. 505-510.

[25] S. Zhu, Z. Zhou, D. Zhang, C. Jin and Z. Li, "Design and Synthesis of Delivery System Based on SBA-15 with Magnetic Particles Formed in Situ and Thermo-Sensitive P-Napalm as Controlled Switch," Microporous and Mesoporous Materials, Vol. 106, No. 1-3, 2007, pp. 56-61. doi:10.1016/j.micromeso.2007.02.027

[26] Z. Zhou, S. Zhu and D. Zhang, "Grafting of Thermo-Responsive Polymer inside Mesoporous Silica with Large Pore Size Using ATRP and Investigation of Its Use in Drug Release," Journal of Materials Chemistry, Vol. 17, No. 23, 2007, pp. 2428-2433. doi:10.1039/b618834f

[27] A. Sousa and E. M. B. Sousa, "Influence of Synthesis Temperature on the Structural Characteristics of Mesoporous Silica," Journal of Non-Crystalline Solids, Vol. 352, No. 32-35, 2006, pp. 3451-3456. doi:10.1016/j.jnoncrysol.2006.03.080

[28] R. G. Sousa, "Structural Characterization of the Gel Thermosensitive Poly( $N$-isopropylacrylamide) and Its Copolymers with Acrylamide," Ph.D. Thesis, Federal University of Minas Gerais, Belo Horizonte, 1997.

[29] A. Sousa, D. A. Maria, R. G. Sousa and E. M. B. Sousa, "Synthesis and Characterization of Mesoporous Silica/ Poly( $N$-isopropylacrylamide) Functional Hybrid Useful for Drug Delivery," Journal of Material Science, Vol. 45, No. 6, 2010, pp. 1478-1486. doi:10.1007/s10853-009-4106-3

[30] T. Kokubo, et al., "Solutions Able to Reproduce in Vivo Surface-Structure Changes in Bioactive Glass-Ceramic A-W," Journal of Biomedical Materials Research, Vol. 24, No. 6, 1990, pp. 721-734. doi: $10.1002 / \mathrm{jbm} .820240607$

[31] T. Higuchi, "Mechanism of Sustained-Action Medication. Theoretical Analysis of Rate of Release of Solid Drugs Dispersed in Solid Matrices," Journal of Pharmaceuticals Science, Vol. 52, No. 12, 1963, pp. 1145-1149. doi:10.1002/jps.2600521210 